Alexander E. P. Heazell • James B. Davis •

Nighat R. Bhatti - Ulises Zanetto

\title{
A case of tubal endometriosis mimicking the appearances of tubal pregnancy at laparoscopy
}

Published online: 31 August 2004

(C) Springer-Verlag Berlin / Heidelberg 2004

\begin{abstract}
Tubal pregnancy is a common gynaecological emergency. Due to the accuracy of transvaginal ultrasonography and serum human chorionic gonadotrophin (hCG) analysis is being managed more frequently by laparoscopy in asymptomatic patients. A case of presumed tubal pregnancy is presented in which the evidence generated by ultrasonography, hCG levels and laparoscopy all suggested tubal pregnancy. However, histological examination showed a tubal endometrioma. This case highlights the importance of histological analysis of all surgical specimens and provides a rare differential diagnosis of tubal mass.
\end{abstract}

Keywords Tubal endometrioma - Tubal pregnancy . Transvaginal ultrasonography $\cdot$ Serum human chorionic gonadotrophin (hCG)

\section{Introduction}

Tubal pregnancy is a common gynaecological emergency, which is due to the accuracy of transvaginal ultrasonography and serum human chorionic gonadotrophin (hCG) analysis is being managed more frequently by laparoscopy in asymptomatic patients. A case of presumed tubal pregnancy is presented in which the evidence generated by ultrasonography, hCG levels and laparoscopy all suggested tubal pregnancy is presented.

\footnotetext{
A. E. P. Heazell $(\bullet)$ J. B. Davis · N. R. Bhatti

Department of Obstetrics and Gynaecology,

City Hospital,

Dudley Road, Birmingham, B18 7QH, UK

e-mail: alex_heazell@talk21.com

Tel.: +44-121-5074042

Fax: +44-121-5075467

U. Zanetto

Department of Histopathology,

City Hospital,

Birmingham, UK
}

\section{Case report}

A 37-year-old parous woman presented to the gynaecology service with 6-week amenorrhea, a positive urine pregnancy test, a single episode of light vaginal bleeding and lower abdominal pain. In addition to a normal vaginal delivery at term, the patient also had a history of two first trimester miscarriages. She had no significant gynaecological history and no known risk factors for tubal pregnancy. On examination, there was mild tenderness in the right iliac fossa; the cervical os was closed, and there was no adenexal tenderness or cervical excitation.

Ultrasound scan showed a thickened endometrium $(17 \mathrm{~mm})$ with no evidence of an intrauterine gestation sac. A $2.2 \mathrm{~cm}$ mass was seen adjacent to the right ovary, with some associated free fluid. A serum hCG was 3,485 iu/l. In accordance with the RCOG guideline (RCOG, 2001), the patient was counselled as to the possibility of a tubal pregnancy, and consented to a laparoscopy, salpingectomy and laparotomy if necessary.

At laparoscopy, a $2 \mathrm{~cm}$ bluish coloured mass was noted in the isthmic portion of the right fallopian tube; this was surrounded by adhesions (Fig. 1). There was a small amount of blood in the Pouch of Douglas, and the left tube had normal appearances. A diagnosis of right tubal pregnancy was made. A decision was made to proceed to laparotomy in view of the close proximity of the mass to the uterus - a right partial salpingectomy was performed. The patient made an uncomplicated recovery.

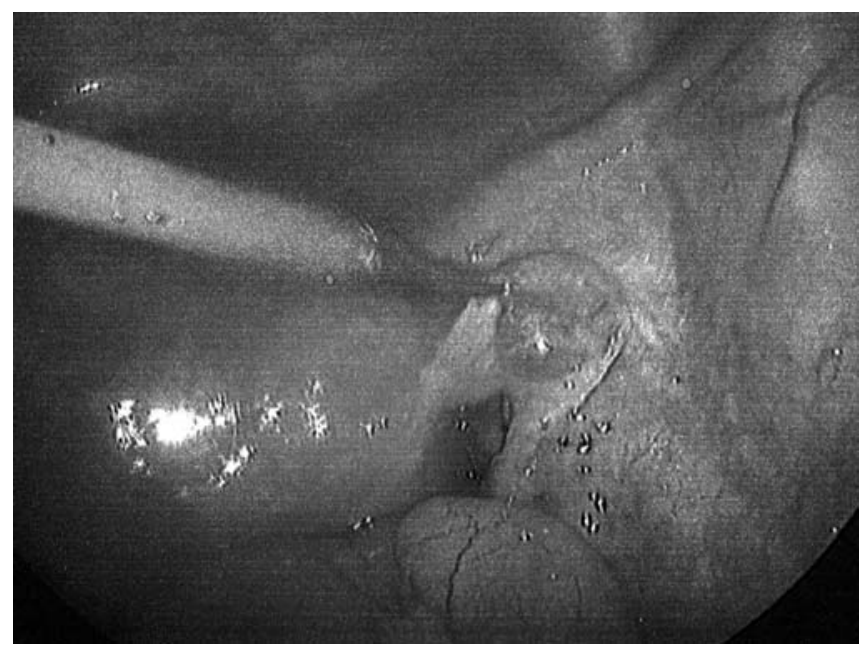

Fig. 1 Laparoscopic appearances of lesion in right tube 


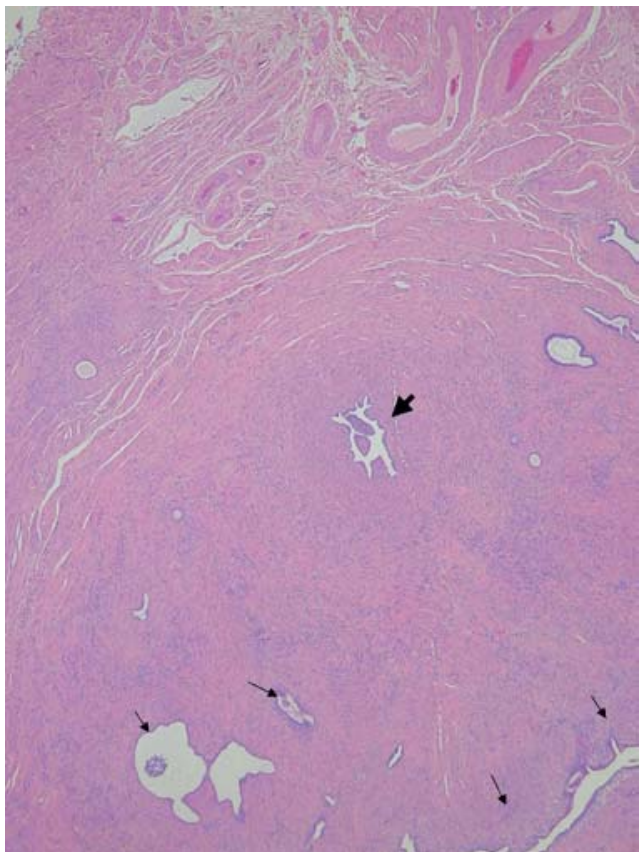

Fig. 2 Low power view $(\times 25)$ of lesion in right tube. Endometrial glands are marked by arrows

Histology revealed a $21 \times 15 \times 15-\mathrm{mm}$ mass in the specimen. Sections showed that the fallopian tube lumen was reduced, and there were aggregates of glands, which had an appearance typical of endometrium. There was also decidualisation of the stroma within the muscle wall-this was in keeping with endometriosis. There was no evidence of trophoblastic tissue (Fig. 2). Ten days post-laparotomy, transvaginal ultrasound scan revealed a small amount of fluid in the endometrial cavity and thickened endometrium $(17 \mathrm{~mm})$ with no evidence of a gestation sac. At this point, the serum hCG had fallen to $175 \mathrm{iu} / \mathrm{l}$, and a diagnosis of failing intrauterine pregnancy was made.

\section{Discussion}

Ectopic pregnancy is a common gynaecological emergency, which with the advent of transvaginal ultrasound and rapid, accurate serum hCG assays is more often managed in asymptomatic, haemodynamically stable patients. Endometriosis is also a common gynaecological disorder, with a prevalence between $43-45 \%[4,5]$; it is usually asymptomatic, but may cause pelvic pain and infertility [5]. The cause of endometriosis is unknown, and it is also associated with tubal damage [4]. In animal studies, species that do not develop endometriosis do not develop ectopic pregnancy [3].

Of all tubal pregnancies, $28 \%$ affect the isthmic region of the tube [2]. In $25 \%$ of cases, the macroscopic appearances of endometriosis are hardened cicatrial lesions of purplish-blue colour in the isthmo-ampullary region of the tube [1]. Given the reported association between endometriosis, tubal dysfunction and tubal pregnancy, and the similarities in macroscopic appearances of both lesions, it is important to be aware of the differential diagnosis of a tubal lesion prior to its excision and the necessity for histological examination of the surgical specimen.

\section{References}

1. Cioltei A, Tasca L, Titiriga L, Maakaron G, Calciu V (1979) Nodular salpingitis and tubal endometriosis II. Diagnosis and differential diagnosis. Acta Eur Fertil 10:147-160

2. Douglas CP (1963) Tubal ectopic pregnancy. Br Med J 2:838841

3. Hunter RHF (2002) Tubal ectopic pregnancy: a patho-physiological explanation involving endometriosis. Hum Reprod 17: 1688-1691

4. Liu DTY, Hitchcock A (1986) Endometriosis: its association with retrograde menstruation, dysmenorrhoea and tubal pathology. Br J Obstet Gynaecol 93:859-862

5. Winkel CA (2003) Evaluation and management of women with endometriosis. Obstetr Gynaecol 102:397-407 